\title{
La conduite de justification: un savoir à transposer des usages
}

\author{
Marie-Hélène Forget \\ Université du Québec à Montréal, Canada
}

Article reçue le 4 Août 2014, a accepté 28 Décembre 2014, version finale reçue le 28 Janvier 2015

DOI: http://dx.doi.org/10.5565/rev/jtl3.588

\begin{abstract}
Resumée
Cet article découle d'une recherche doctorale ${ }^{\mathrm{i}}$ ayant porté sur l'apprentissage de la justification en classe de français. Il vise à faire état de la question de la transposition didactique de la notion de justification proposée dans les programmes d'études du primaire et du secondaire québécois, à mieux définir la justification et à mieux la distinguer d'autres discours apparentés, ce, par une recension d'écrits du domaine. L'article vise également, en se basant sur des travaux majeurs en sociolinguistique, à remettre en question la conception véhiculée dans des classes de français du secondaire selon laquelle la justification correspond à une structure textuelle prototypique. L'article propose enfin une transposition didactique axée sur la variabilité des usages langagiers et sur la pluralité des conduites de justification en soulignant l'intérêt d'une telle transposition pour soutenir le développement de compétences justificatives qui soient utiles dans toutes les sphères de la vie.
\end{abstract}

Mots clés: Justification, discours, situation, transposition didactique, sociolinguistique

\begin{abstract}
This article arises from a doctoral research examining the learning of justification in a French class. It aims to reflect on the didactical transposition of the concept of justification suggested in Québec's curriculum for primary and secondary schools, to further define this concept, and to distinguish it from other similar discourses through a review of the literature in the fields of linguistics and pedagogy. This article also aims to question the notion conveyed in secondary level French classes that justification corresponds to a prototypical textual pattern, based on major sociolinguistics research. Finally, we propose a didactical transposition focused on the multiplicity of language uses by underlining the use of this transposition to support the development of justifying skills that are useful in all areas of life.
\end{abstract}

Keywords: Justification, discourse, situation, didactic transposition; sociolinguistic

\section{Resumen}

Este artículo es producto de una investigación doctoral sobre el aprendizaje de la justificación en clases de francés. Pretende hacer valer el asunto de la transposición didáctica de la noción de justificación propuesta en los programas de estudios primarios y secundarios de Quebec; proponer una mejor definición de la misma; y distinguirla de otros discursos semejantes, por medio de una reseña de escritos en lingüística y en didáctica. Tomando como base algunos estudios importantes de sociolingüística, el artículo también pretende cuestionar la concepción vehiculada en las clases de francés de secundaria según la cual la justificación corresponde a un esquema textual prototípico. Finalmente, el artículo propone una transposición didáctica centrada en la variabilidad de los usos del lenguaje y en la pluralidad de la justificación, remarcando lo interesante de tal propuesta para mantener el desarrollo de competencias justificativas, que serán útiles en todas las esferas de sus vidas.

Palabras clave: Justificación, discurso, situación, transposición didáctica, sociolingüístico 


\section{Aperçu de la problématique}

En 2004, dans la foulée de la réforme de l'éducation amorcée à l'ordre primaire, le ministère québécois de l'Éducation, du Loisir et du Sport (MÉLS) prescrit un nouveau programme de français langue d'enseignement pour le $1^{\text {er }}$ cycle du secondaire ${ }^{\mathrm{ii}}$ (MÉLS, 2004) dans lequel des savoirs à enseigner sont ajoutés, dont la notion de justification présentée comme une «sensibilisation à l'argumentation» (MÉLS, 2004, p. 110). Cinq ans plus tard, le ministère introduit une épreuve nationale d'écriture à la fin du $1^{\text {er }}$ cycle du secondaire (MÉLS, 2009a) qui évalue la compétence scripturale de justification. Dès lors, la maitrise ${ }^{\text {iii }}$ de cette notion apparait comme une priorité pour les instances ministérielles.

Ces nouvelles orientations tranchent avec les pratiques habituelles des enseignants qui, en écho au programme précédent (MEQ, 1995), concentraient surtout le travail sur l'argumentation dans les dernières années du secondaire. Aussi, des enseignants ont émis certains doutes à propos des capacités des élèves du $1^{\text {er }}$ cycle à entamer le développement de compétences liées à l'argumentation. Enfin, si l'on s'en tient aux instructions officielles, la justification demeure difficile à définir. Dans le programme du $1^{\text {er }}$ cycle (MÉLS, 2004), on indique que pour justifier l'élève « apprend à circonscrire et à mettre en évidence les raisons qui orientent ou fondent une position» (p. 109-110). Dans le programme du $2^{\mathrm{e}}$ cycle secondaire (MÉLS, 2009b), la justification et l'argumentation semblent se confondre étant toutes deux associées aux notions de thèse, d'opinion et d'argument. Ailleurs encore, on distingue mal la justification de l'explication en parlant pour les deux modes de discours de « rapport de causalité » (Ibid., p. 65).

Pour mieux distinguer l'argumentation, l'explication et la justification, la Progression des apprentissages au secondaire (MÉLS, 2011) spécifie que : «Contrairement à l’explication, elle [la justification] ne vise pas à faire comprendre en rendant visibles les relations de causalité entre des éléments [...]. La justification ne fait qu'expliciter à postériori la démarche cognitive (le raisonnement) qui a conduit à l'expression du propos » (p. 21). Quant à la distinction entre l'argumentation et la justification, on y précise que la justification « ne cherche pas à convaincre le destinataire d'adopter une thèse sur un sujet controversé où s'affrontent deux thèses opposées » (p. 21), mais vise plutôt à faire valoir la légitimité d'un propos ou d'une réponse à une question. On indique par ailleurs que « la situation de communication peut faire basculer une justification en une argumentation» (p. 21) sans plus de précisions. Ainsi, des flous persistent dans les 
instructions officielles quant aux caractéristiques propres à la justification entrainant ambigüités et confusions chez le corps enseignant du $1^{\mathrm{er}}$ cycle du secondaire néanmoins responsable de son enseignement. Trois questions ont alors été formulées par des enseignants:

1- qu'est-ce que justifier signifie dans le cadre de la classe de français ?;

2- quelles attentes peut-on réalistement fixer en ce qui concerne les capacités des élèves de cet âge à justifier leurs propos ?;

3- comment enseigner la justification au $1^{\mathrm{er}}$ cycle du secondaire ?

Pour soutenir les milieux de pratique et contribuer à l'avancement de la réflexion didactique autour de cette notion relativement nouvelle, cet article propose, en appui sur une recherche réalisée dans trois classes de français du $1^{\text {er }}$ cycle du secondaire québécois, des éléments de réponse à la première et à la troisième questions. D'abord, nous clarifierons, par une recension d'écrits du domaine, la définition de la notion de justification et la distinguerons mieux d'autres discours apparentés. Puis, après avoir fait ressortir le caractère variable et pluriel de la justification en fonction des contextes (Porquier et Py, 2004), nous suggèrerons de procéder à une transposition didactique (Chevallard, 1985/1991) appuyée sur les pratiques quotidiennes et de référence (Blanchet, 2011; Martinand, 1989). Nous croyons qu'une telle transposition favoriserait le développement de compétences justificatives utiles pour toutes les sphères de la vie des élèves.

\section{Qu'est-ce que justifier?}

Pour bien des spécialistes de la didactique, la justification correspond à l'opération de base de l'argumentation (Auriac-Peyronnet, 2001; de Pietro, Érard, et Kaneman-Pougatch, 1997; GarciaDebanc, 1996; Golder, 1996; Gombert, 1998, 2003). Par ailleurs, certains autres l'associent plutôt à une conduite démonstrative (Chartrand, 2008, 2013) ou ayant une valeur explicative (Miled, 1998). Voyons d'abord, d'un point de vue linguistique, ce qui la distingue de l'explication, de la démonstration et de l'argumentation avant de préciser ce qu'on entend par « justifier » en classe de français.

Justifier vs expliquer. Expliquer sert à modifier l'état des connaissances d'un interlocuteur, à lui faire comprendre les causes d'un fait ou d'un phénomène que l'on peut observer (Duval, 1992; Fasel-Lauzon, 2014). L'énonciateur d'une explication se place alors en position de témoin des faits dont il rend compte. S'il n'est pas l'agent du phénomène expliqué, il 
est néanmoins réputé apte à en fournir l'explication, ce qui le place en position « haute » par rapport à ses interlocuteurs (Borel, 1981). Dans le cas d'une justification, l'énonciateur soumet à l'examen des interlocuteurs les raisons qui l'ont mené à la position qu'il adopte. Ce faisant, il est placé en position «basse » par rapport à ses interlocuteurs qui jouent alors un rôle d'évaluateur. Justifier reste alors une « tentative d'accroître le crédit accordé par l'interlocuteur à un jugement donné, [d'où sa] valeur plus proprement argumentative » (Apothéloz et Brandt, 1992, p. 88).

La justification comme l'explication servent à répondre à des questions de type «pourquoi » (Adam, 1996; Dufour, 2008; Duval, 1992; Gombert, 2003; Grize, 1996; Plantin, 2005) : «pourquoi affirmes-tu cela? » dans le cas d'une justification, « pourquoi est-ce ainsi? » dans le cas d'une explication. Adam (1992, 2005), Duval (1992) et Zakhartchouk (2001) font également observer que la justification et l'explication sont, sur le plan textuel, tout à fait semblables : on présente d'abord ce qui fait l'objet d'une justification ou d'une explication, puis on introduit, par un connecteur de type «parce que », les raisons qui appuient ou motivent la position (si l'on justifie) ou les causes qui expliquent le phénomène (si l'on explique) avant de conclure.

Justifier vs démontrer. Démontrer c'est prouver que la conclusion d'un raisonnement (logique) est vraie. L'énonciateur qui procède à une démonstration soumet à l'examen de ses interlocuteurs chaque étape du raisonnement qu'il a suivi pour parvenir à sa conclusion. La démonstration demande à ce que le raisonnement tout autant que les éléments de preuve utilisés soient valides, c'est-à-dire que le raisonnement soit complet et logiquement organisé, et que les éléments de preuve soient admis et justes par rapport au modèle théorique (théorèmes, lois, règles) de référence (Buty et Plantin, 2008; Duval, 1992; Grize, 1996; Plantin, 2005). Par exemple, pour être recevable, un raisonnement grammatical doit faire appel à un même modèle théorique (ex: la grammaire nouvelle), s'appuyer sur les bonnes règles et manipulations syntaxiques, enchainer les étapes du raisonnement de manière logique tout en étant complet.

Dans le cas de la justification, ce n'est pas tant la validité que la pertinence du raisonnement de l'énonciateur qui est soumis à l'examen (Borel, 1981; Perelman, 1961). Cette pertinence n'est pas nécessairement tributaire d'un appui sur un modèle théorique stable (Zakhartchouk, 2001) : la justification peut faire appel à des connaissances encore partielles et en construction, ce qui est le cas des connaissances des élèves à l'école. La justification peut 
également faire appel à des valeurs, à des principes, à des préférences, à des indices. Les raisons invoquées et leur enchainement peuvent aussi changer en fonction du contexte et des interlocuteurs, ce qui n'est pas le cas pour la démonstration. En somme, on justifie ce qui reste encore discutable et peut encore être nuancé, voire rejeté (Borel, 1981).

Justifier vs argumenter. Pour comparer enfin la justification à l'argumentation, il faut d'abord souligner que deux conceptions d'argumentation cohabitent actuellement en éducation : l'argumentation à caractère rhétorique, largement enseignée en classe de français au $2^{\mathrm{e}}$ cycle du secondaire québécois, et l'argumentation euristique, qui tarde un peu à s'implanter dans les mœurs éducatives en tant que savoir à enseigner, même si elle est très présente dans les échanges et les discussions scolaires et sociales (Buty et Plantin, 2008; Nonnon, 1996).

L'approche dite «rhétorique» de l'argumentation (Thyrion, 1997) s'attarde à l'effet persuasif d'abord et avant tout : on cherche à convaincre, on s'oriente vers l'action immédiate. Cette approche suppose un objet de débat préalablement construit et des arguments visant à obtenir l'adhésion des interlocuteurs à une thèse. S'il y a bel et bien une dimension dialogique dans l'argumentation rhétorique, la prise en compte de son destinataire sert à anticiper les arguments de ce dernier afin de s'en servir ou de mieux les réfuter. Les procédés d'opposition, de concession ou de réfutation abondent et servent toujours à conserver une position offensive. Si l'énonciateur laisse entendre d'autres voix que la sienne, des citations d'experts par exemple, ce n'est que pour mieux mettre sa position en valeur. Dans ce type d'argumentation, changer d'avis est perçu comme un échec ou à tout le moins comme une contradiction.

L'argumentation à caractère euristique vise l'élaboration d'idées ou la construction de savoirs dans le cadre d'échanges qui sont motivés et structurés par le besoin à combler et non par la recherche à tout prix de l'adhésion à une thèse (Nonnon, 1996; Thyrion, 1997). Si les matériaux et procédés utilisés sont semblables à ceux des discours rhétoriques (thèse, arguments, concession, réfutation, etc.), sa finalité n'est pas celle de persuader, mais de négocier une position recevable ou admissible qui fera idéalement, mais pas obligatoirement ou immédiatement, consensus. Le fait de gagner ou de perdre prend beaucoup moins d'importance, l'argumentation produisant des conclusions provisoires, négociables et ouvertes (Grize, 1996). Elle sert à explorer les idées, à trouver sa position et à la fonder, à comprendre et à apprendre. 
L'argumentation peut ainsi se concevoir en tant que continuum allant de la persuasion orientée vers un résultat pratique à la dialectique orientée vers la construction des connaissances (Thyrion, 1997). Si les situations argumentatives impliquent toujours une controverse, celle-ci va refléter le continuum argumentatif, selon que l'énonciateur veut à tout prix persuader ou au contraire qu'il souhaite discuter (Buty et Plantin, 2008; Duval, 1992; Golder, 1996; Nonnon, 1996; Plantin, 2005). Pour argumenter il faut recourir à des arguments crédibles et pertinents pour les parties puisque c'est la valeur de vraisemblance qui prévaut. L'argumentation articule plusieurs points de vue qui seront d'abord justifiés (Gombert, 2003), puis négociés ou réfutés selon l'intention poursuivie. La justification se trouve alors au cœur de l'argumentation dans la mesure où elle en est le point de départ (Gombert, 1998; van Eemeren et Grootendorst, 1996).

Justifier. À tout reprendre, la justification est « un acte qui a pour visée de faciliter ou de causer l'acceptation de son intention par l'interlocuteur » (Garcia-Debanc, 1996, p. 114), c'est-àdire de rendre un propos ou un comportement légitime aux yeux de l'interlocuteur (Borel, 1981; Brandt, 1992). Une justification survient lorsqu'une question comme «Pourquoi affirmes-tu cela ?» (Chartrand, 2013, n.p.) ou « Pourquoi agis-tu comme cela ? » est posée de façon explicite ou implicite par un interlocuteur qui souhaite que l'énonciateur lui fournisse les raisons qui fondent son propos ou son comportement. La justification concerne alors ce que pense, croit ou suggère une personne à propos d'une chose, ou bien ce qui la motive à agir comme elle le fait. L'énonciateur est alors placé en position basse par rapport à son interlocuteur, réputé apte à juger de la légitimité de ses actes. Ainsi, la justification se distingue d'autres conduites par la perspective subjective qu'elle offre (Fasel-Lauzon, 2014). En effet, elle donne accès au regard que porte son énonciateur sur un objet, c'est-à-dire au raisonnement l'ayant mené à sa conclusion, aux liens qu'il a établis entre des indices, aux raisons ou aux motivations qui soustendent sa position. En somme, on justifie ce qui est « vrai » ou «pertinent » pour soi, et qu'il nous reste encore à faire valoir aux yeux des autres.

Justifier en classe de français. En classe de français, deux types d'assertions doivent généralement être justifiées : des réponses à des questions et des positions (avis, opinions, préférences). Quand l'élève cherche à justifier une réponse à une question, il doit montrer pourquoi sa réponse est vraie ou valide (justifier l'accord d'un verbe ou sa compréhension d'un texte par exemple). L'élève devra alors recourir aux règles, aux connaissances et aux éléments 
qui sont valides (les manipulations syntaxiques requises) ou pertinents (des indices dans un texte) dans la situation. Il va rendre compte du raisonnement qu'il a effectué ou des liens qu'il a établis pour parvenir à sa réponse. Puisque l'élève cherche en quelque sorte à prouver ce qu'il avance en fournissant ce qu'il convient d'appeler des preuves, mais que ses connaissances sont en construction, on peut considérer qu'il utilise la justification dans une visée démonstrative (Chartrand, 2013). Un désaccord ou une objection de la part de l'interlocuteur pourra faire basculer la justification en argumentation puisqu'une controverse autour d'un savoir en construction aura alors émergé de l'échange.

Quand l'élève cherche à légitimer sa position sur un sujet, il ne cherche pas nécessairement à convaincre son destinataire d'adopter son point de vue, il cherche plutôt à le convaincre de l'acceptabilité de sa position (van Eemeren et Grootendorst, 1996). Pour ce faire, il va la justifier, c'est-à-dire qu'il va l'étayer à l'aide des connaissances, des motivations, des valeurs ou des raisons qui la fondent. C'est le cas par exemple lorsque l'élève est appelé à donner son avis sur la qualité d'un roman ${ }^{\mathrm{v}}$ ou à faire valoir son interprétation de l'extrait d'un texte. Bien qu'il n'y ait pas de controverse de prime abord, il est possible qu'une bascule ait lieu si un interlocuteur s'inscrit en faux par rapport à la position présentée par l'énonciateur. Par exemple, l'interprétation qu'un élève fait de l'extrait d'un roman peut être plausible, mais ne pas être partagée par ses interlocuteurs qui la remettront alors en question. Un tel type de justification a une visée $\operatorname{argumentative~}^{\mathrm{vi}}$ puisqu'il vise à faire valoir la pertinence d'une opinion, la vraisemblance d'une interprétation ou la légitimité de ses gouts, de ses préférences ou de ses valeurs.

Un troisième type d'assertion, la proposition, est suggéré par Masseron (2003) qui soutient, en appui sur les travaux de J.-M. Adam, que l'acte directif du conseil ou de la recommandation implique une justification, puisqu'il s'agit de fournir au destinataire ou à l'interlocuteur les raisons valables d'agir dans le sens de la proposition. Pour faire valoir une proposition, l'énonciateur va se servir d'éléments d'étayage de manière stratégique afin d'inciter son destinataire à suivre son conseil. Il devra alors tenir compte des connaissances, des critères, des valeurs, des principes, des émotions, des gouts de son destinataire afin de l'inciter à agir. Une très bonne connaissance de ce dernier apparait incontournable. Encore ici, il ne s'agit pas d'une situation controversée, mais il convient de considérer ce type de justification comme ayant une 
visée argumentative dans la mesure où l'intention de l'énonciateur est orientée vers l'action qu'il souhaite voir accomplie par son destinataire (Thyrion, 1997).

\section{Quelle transposition didactique en proposer?}

$\mathrm{Au}$ secondaire québécois, les enseignants ont tendance à présenter les discours comme des structures prototypiques et à mettre l'accent sur leurs caractéristiques textuelles et linguistiques (Forget, 2012). Cette transposition didactique interne ${ }^{\text {vii }}$ (Chevallard, 1985/1991; Gauvin et Boivin, 2012), c'est-à-dire la transposition qu'effectue l'enseignant en classe, véhicule sciemment ou pas l'idée selon laquelle les discours correspondent à des objets stables et uniformes. Or, s'il est vrai que la justification possède des caractéristiques textuelles relativement stables (Adam, 1992, 1996, 2005) qu'il est utile de connaitre pour optimiser la maitrise de ce discours (Brassart, 1990), les usages scolaires et sociaux des discours montrent leur caractère pluriel, variable, métissé, évolutif, complexe, culturellement et contextuellement marqué (Blanchet, 2007; de Robillard, 2007; Porquier et Py, 2004). En effet, l'énonciateur d'une justification adapte ce qu'il dit ou écrit, ainsi que la manière de le dire ou de l'écrire, aux contraintes énonciatives, pragmatiques et socioculturelles inhérentes à la situation du moment : le contenu et la forme d'une justification dépendent donc du contexte (Porquier et Py, 2004).

Aussi, comme la justification se met en place très tôt dans la vie du jeune enfant qui l'utilise et s'en approprie les règles d'usage dans une multitude de situations d'interaction, dans la famille et la sphère sociale ainsi qu'à l'école (Buty et Plantin, 2008; Dunn et Munn, 1987; Garcia-Debanc, 1996; Goetz et Shatz, 1999; Golder, 1996; Gombert, 2003; Kyratzis, ShuqumRoss, et Koymen-Bahar, 2010), les élèves qui entrent au secondaire amènent avec eux un savoir d'expérience de cette conduite (Forget, 2014) qui les rend aptes à justifier dans une panoplie de situations d'interactions orales plutôt informelles. Il leur reste néanmoins, si l'on se fie aux recherches du domaine (par ex. Garcia-Debanc, 1994; Golder, 1996), à construire des connaissances et à développer des compétences qui les rendent mieux aptes à justifier dans des situations plus formelles, à l'oral comme à l'écrit. Mais, parce qu'elle élude le caractère variable et pluriel de la conduite et passe sous silence les capacités des élèves à conduire des justifications dans diverses situations, on est conduit à reconsidérer la transposition didactique interne actuellement proposée en classe de français au secondaire québécois. 
Pour enrichir leurs potentialités langagières (Sabatier, 2008), la transposition didactique interne (Chevallard, 1985/1991; Gauvin et Boivin, 2012) du discours de justification devrait permettre de mieux comprendre comment les discours varient en fonction des situations et de développer des pratiques scolaires et sociales de justification (Martinand, 1989) plus formelles. Une telle transposition didactique profiterait d'une stratégie d'intervention en deux étapes : la première étape consiste à inventorier les déjà-là des élèves en matière de justification. La seconde étape vise à élargir les potentialités discursives des élèves en les plaçant dans des situations requérant la production d'un discours justificatif plus formel (oral ou écrit), afin de soutenir l'appropriation de connaissances et de comportements cognitifs et langagiers plus appropriés à de telles situations.

Faire l'inventaire des déjà-là. S'intéresser aux déjà-là offre une occasion de valoriser ce que les élèves amènent avec eux et de mieux connaitre leurs besoins d'apprentissage (Reuter, 2001). Pour procéder à l'inventaire des déjà-là des élèves en matière de justification, on les invite à évoquer avec beaucoup de précisions le souvenir d'une situation ayant, de leur point de vue, engendré des conduites de justification. On prend soin de solliciter des récits de différentes (à l'école, dans la famille, entre amis, etc.) de manière à faire ressortir l'étendue des usages et des formes possibles de ce discours. Le tableau suivant indique les aspects pour lesquels il est intéressant de recueillir des informations.

Tableau 1. Informations à recueillir à propos des diverses situations de justification

\begin{tabular}{lll}
\hline \multicolumn{1}{c}{ Situations } & \multicolumn{1}{c}{ Conduites } & \multicolumn{1}{c}{ Actions de l'énonciateur } \\
\hline Circonstances & Objet de la justification & Éléments à mettre en valeur/à \\
Lieu (institution et endroit) & Spontanée/préparée & éviter \\
Interlocuteurs (rôle, statut, & Informelle/formelle & Stratégie(s) mise(s) en place \\
attitudes et dispositions) & Orale/écrite (à préciser) & Préparation (oui/non? Comment?) \\
Énonciateur (rôle, statut, état & En direct/en différé (implications) & Manières de s'ajuster \\
émotif) & En langue première/seconde & Choix des raisons/des mots \\
Intentions et enjeu & Organisation du texte/genre & Difficultés rencontrées/solutions \\
Déroulement et dénouement & Construite seul ou à plusieurs & Indices de réussite/échec \\
Etc. & Etc. & Etc. \\
\hline
\end{tabular}

Comparer les situations. Les situations détaillées par les élèves sont ensuite comparées de manière 1- à définir la conduite de justification en dégageant ce qui est commun à toute conduite de justification quelle que soit la situation (ex : sa finalité); 2- à mettre en relief ce qui varie en fonction des contextes (ex : le choix des raisons à l'appui en fonction de l'interlocuteur); 3- à 
réajuster, au besoin, certaines conceptions erronées de la justification ou inopérantes (ex: clarifier la distinction entre expliquer et justifier).

L'acquisition de nouvelles connaissances. Pour guider les élèves dans l'élargissement de leurs potentialités discursives, l'enseignant prendra soin de proposer à ses élèves des situations d'apprentissage de la justification qui demandent l'acquisition de nouvelles connaissances et la mise en œuvre des pratiques scolaires et sociales ${ }^{\text {viii }}$ plus formelles et complexes en s'appuyant sur le déjà-là. Par exemple, recommander à un ami au cours d'un échange téléphonique informel une sortie au cinéma s'avère une pratique potentiellement connue des élèves qui en décriront les caractéristiques assez aisément (en autant qu'ils y soient amenés). Partant de là, l'écriture d'une note critique ou d'une recommandation de lecture destinée aux internautes nécessitent l'apprentissage de la construction d'une représentation juste et claire de son destinataire alors moins connu, l'appropriation des caractéristiques de ces genres formels et davantage de planification en ce qui a trait au contenu du texte.

L'intégration du nouveau aux déjà-là. Mesurer le chemin parcouru valorise les nouveaux acquis, met en lumière l'élargissement des potentialités et aide tout à la fois à percevoir ce qui reste à apprendre, ce que l'ajout du « nouveau » à l'inventaire de départ permet d'apprécier. Cette intégration peut s'effectuer par un retour sur l'analyse comparative des situations en y incluant celle qui vient d'être vécue en classe. De nouveaux objectifs d'apprentissage peuvent alors être fixés.

\section{Conclusion}

Cet article souhaitait contribuer à mieux définir la justification et à la distinguer d'autres discours apparentés que sont l'explication, la démonstration et l'argumentation. Il visait également à suggérer une transposition didactique de ce nouveau savoir à enseigner qui s'appuie sur les usages du quotidien et favorise l'appropriation de pratiques scolaires et sociales de référence (Martinand, 1989). Une telle transposition didactique peut amener les élèves à prendre conscience de leurs pratiques langagières et à constater l'importance d'élargir leurs potentialités en matière de justification. Elle peut également, par le recours à des activités réflexives sur les pratiques langagières, soutenir le développement de compétences langagières plus solides. Une telle transposition didactique favoriserait enfin un certain «effet passerelle» en permettant à 
l'élève de rattacher « de l'inconnu au connu, du nouveau à de l'existant » (Reuter, 2001, p. 20). Quand on sait que les déjà-là s'articulent inévitablement aux savoirs enseignés et peuvent soutenir ou entraver leur apprentissage selon qu'ils entrent en résonnance ou en dissonance avec les attentes scolaires (Penloup, 2005; Reuter, 2001; Reuter et Penloup, 2001), en tenir compte apparait aussi bénéfique qu'essentiel. En somme, c'est bien d'une transposition didactique axée sur l'apprenant, ses pratiques et ses besoins (Penloup, 2005) que cette proposition contribuerait à développer.

\section{Références bibliographiques}

Adam, J.-M. (1992). Les textes: types et prototypes. Récit, description, argumentation, explication et dialogue. Paris: Nathan.

Adam, J.-M. (1996). L'argumentation dans le dialogue. Langue française, 112(1), 31-49. http://dx.doi.org/10.3406/lfr.1996.5359

Adam, J.-M. (2005). La linguistique textuelle : introduction à l'analyse textuelle des discours. Paris: Armand Colin.

Apothéloz, D., et Brandt, P.-Y. (1992). Relation d'étayage : Justification et/ou explication? Travaux du Centre de recherches sémiotiques, 60, 55-88.

Auriac-Peyronnet, E. (2001). The impact of oral training on argumentative texts produced by tenand eleven-year-old children: Exploring the relation between narration and argumentation. European Journal of Psychology of Education, 16(2), 299-317. http://dx.doi.org/10.1007/bf03173031

Blanchet, P. (2007). Quels «linguistes» parlent de quoi, à qui, quand, comment et pourquoi? Pour un débat épistémologique sur l'étude des phénomènes linguistiques. Dans P. Blanchet, L.J. Calvet et D. de Robillard (dir.), Un siècle après Saussure: la linguistique en question (p. 229-294). Paris: L'Harmattan.

Blanchet, P. (2011). Les transpositions didactiques. Dans P. Blanchet et P. Chardenet (dir.), Guide pour la recherche en didactique des langues et des cultures: Approches contextualisées (p. 197-202). Paris: Éditions des archives contemporaines.

Borel, M.-J. (1981). Donner des raisons. Un genre de discours : l'explication. Revue européenne de sciences sociales, 19(56), 37-68.

Brandt, P.-Y. (1992). Les organisations raisonnées: Justifier, argumenter, étayer... Travaux $d u$ Centre de recherches sémiotiques, 60, 89-103.

Brassart, D. (1990). Le développement des capacités discursives chez l'enfant de 8 à 12 ans: le discours argumentatif écrit. Revue française de pédagogie, 90, 31-41. http://dx.doi.org/10.3406/rfp.1990.1394

Buty, C., et Plantin, C. (2008). Argumenter en classe de sciences : du débat à l'apprentissage. Lyon: INRP.

Chartrand, S.-G. (2008). Progression dans l'enseignement du français langue première au secondaire québécois: Répartition des genres textuels, des notions, des stratégies et des 
procédures à enseigner de la lre à la 5e secondaire. Québec: Les publications Québec français.

Chartrand, S.-G. (2013). Enseigner à justifier ses propos de l'école à l'université. Correspondance, 19(1). Repéré à http://correspo.ccdmd.qc.ca/Corr19-1/2.html

Chartrand, S.-G., Émery-Bruneau, J., Sénéchal, K., et Riverin, P. (2013). Caractéristiques de 50 genres pour développer les compétences langagières en français au secondaire québécois. Québec: Didactica.

Chevallard, Y. (1985/1991). La transposition didactique. Du savoir savant au savoir enseigné. Grenoble: La Pensée sauvage.

de Pietro, J.-F., Érard, S., et Kaneman-Pougatch, M. (1997). Un modèle didactique du" débat": de l'objet social à la pratique scolaire. Enjeux, 39-40, 100-129.

de Robillard, D. (2007). La linguistique autrement: altérité, expérienciation, réflexivité, constructivisme, multiversalité: en attendant que le Titanic ne coule pas. Dans P. Blanchet, L.-J. Calvet et D. de Robillard (dir.), Un siècle après Saussure: La linguistique en question (p. 81-228). Paris: L'Harmattan.

Dufour, M. (2008). Argumenter: cours de logique informelle. Paris: Armand Colin.

Dunn, J., et Munn, P. (1987). Development of justification in disputes with mother and sibling. Developmental Psychology, 23(6), 791-798. http://dx.doi.org/10.1037//00121649.23.6.791

Duval, R. (1992). Argumenter, démontrer, expliquer : continuité ou rupture cognitive? Petitx, 31, 37-61.

Fasel-Lauzon, V. (2014). Comprendre et apprendre dans l'interaction. Les séquences d'explication en classe de français langue seconde. Bruxelles: Peter Lang.

Forget, M.-H. (2012). Apprendre à justifier ses propos au secondaire: quels savoirs enseigner, quelle progression proposer? Dans J.-L. Dumortier, J. Van Beveren et D. Vrydaghs (dir.), Curriculum et progression en français. Actes du lle colloque de l'AIRDF (p. 177-191). Namur: Presses universitaires de Namur.

Forget, M.-H. (2014). Pratiques d'écriture de justifications d'élèves plurilingues du ler cycle du secondaire. Entre savoirs d'expérience et apprentissages en classe de français. Thèse de doctorat inédite, Université de Sherbrooke, Sherbrooke.

Garcia-Debanc, C. (1994). Apprendre à justifier par écrit une réponse : analyses linguistiques et perspectives didatiques. Pratiques, 84, 5-40.

Garcia-Debanc, C. (1996). Apprendre à justifier à l'école et au collège : ruptures ou continuité? Dans J. David et S. Plane (dir.), L'apprentissage de l'écriture de l'école au collège (p. 105-130). Paris: Presses universitaires de France.

Gauvin, I., et Boivin, M.-C. (2012). La théorie de la transposition didactique : un outil conceptuel pour décrire les savoirs grammaticaux élaborés en classe par les élèves. Journal of Teaching \& Learning Language \& Literature, 5(30), 10-24.

Goetz, P.-J., et Shatz, M. (1999). When and how peers give reasons: Justifications in the talk of middle school children. Journal of child language, 26(3), 721-748. http://dx.doi.org/10.1017/s0305000999003980

Golder, C. (1996). Le développement des discours argumentatifs. Neuchâtel: Delachaux et Niestlé. 
Gombert, A. (1998). Comment les rédacteurs de 10 à 13 ans se justifient-ils et argumentent-ils? Rôle du thème rédactionnel, de l'opinion consensuelle et de la thèse défendue. Thèse de doctorat inédite, Université de Provenc, Aix-en-Provence.

Gombert, A. (2003). Comment faciliter l'argumentation au primaire. Dans E. Peyronnet (dir.), Je parle, tu parles, nous apprenons: Coopération et argumentation au service des apprentissages (p. 263-274). Bruxelles: De Boeck.

Grize, J.-B. (1996). Logique naturelle et communications. Paris: Presses universitaires de France.

Kyratzis, A., Shuqum-Ross, T., et Koymen-Bahar, S. (2010). Validating justifications in preschool girls' and boys' friendship group talk: implications for linguistic and sociocognitive development. Journal of Child Language, 37, 115-144. http://dx.doi.org/10.1017/s0305000908009069

Martinand, J.-L. (1989). Pratiques de référence, transposition didactique et savoirs professionnels en sciences et techniques. Les sciences de l'éducation pour l'ère nouvelle, 2, 23-29.

Masseron, C. (2003). Conseiller un film d'horreur... justifications et jugements de valeur dans quelques copies de secondes. Pratiques, 117-118, 219-240.

Miled, M. (1998). La didactique de la production écrite en français langue seconde. Paris: Didier Érudition.

Ministère de l'Éducation du Québec (1995). Programme de français : enseignement secondaire. Québec: Gouvernement du Québec.

Ministère de l'Éducation, du Loisir et du Sport (2004). Programme de formation de l'école québécoise : secondaire ler cycle. Québec: Gouvernement du Québec.

Ministère de l'Éducation, du Loisir et du Sport (2009a). Épreuve obligatoire, Français, fin du ler cycle secondaire. Québec: Gouvernement du Québec.

Ministère de l'Éducation, du Loisir et du Sport (2009b). Programme de formation de l'école québécois : Français, langue d'enseignement, secondaire $2 e$ cycle. Québec: Gouvernement du Québec.

Ministère de l'Éducation, du Loisir et du Sport (2011). La progression des apprentissages au secondaire : Français, langue d'enseignement. Québec: Gouvernement du Québec.

Nonnon, E. (1996). Activités argumentatives et élaboration de connaissances nouvelles : Le dialogue comme espace d'exploration. L'argumentation en dialogues, 112, 67-87.

Penloup, M.-C. (2005). Vers une didactique de l'écriture centrée sur l'apprenant et ses pratiques. Dans J. Lafont-Terranova et D. Colin (dir.), Didactique de l'écrit: la construction des savoirs et le sujet-écrivant (p. 81-104). Namur: Presses universitaires de Namur.

Perelman, C. (1961). Jugements de valeur, justification et argumentation. Revue internationale de philosophie, 58(4), 45-55.

Plantin, C. (2005). L'argumentation. Paris: Presses universitaires de France.

Porquier, R., et Py, B. (2004). Apprentissage d'une langue étrangère : contextes et discours. Paris: Didier.

Reuter, Y. (2001). La "prise en compte" des pratiques extrascolaires de lecture et d'écriture: problèmes et enjeux. Repères, 23, 9-31.

Reuter, Y., et Penloup, M.-C. (2001). Présentation. Dans Y. Reuter et M.-C. Penloup (dir.), Les pratiques extrascolaires de lecture et d'écriture des élèves (p. 3-8). Paris: INRP.

Sabatier, C. (2008). Compétence plurilingue et dynamiques d'appropriation langagière. Dans D. Moore et V. Castellotti (dir.), La compétence plurilingue: regards francophones (p. 105126). Berne: Peter Lang. 
Thyrion, F. (1997). L'écrit argumenté : questions d'apprentissage. Louvain-la-Neuve: Peeters. van Eemeren, F. H., et Grootendorst, R. (1996). La nouvelle dialectique. Paris: Editions Kimé. Zakhartchouk, J. M. (2001). Justifiez, expliquez. Pratiques, 111-112, 179-188.

\footnotetext{
${ }^{i}$ Cette thèse a été dirigée par le professeur Olivier Dezutter de l'Université de Sherbrooke (Québec, Canada) et codirigée par la professeure Cécile Sabatier de Simon Fraser University (Colombie-Britannique, Canada).

ii Le secondaire québécois suit les six années de l'école primaire et accueille les élèves à compter de l'âge de 12 ans. Le $1^{\text {er }}$ cycle du secondaire correspond aux deux premières années du cours secondaire qui compte cinq années au total.

iii Ce texte adopte l'orthographe rectifiée.

iv La Progression des apprentissages au secondaire (MÉLS, 2011) est un document complémentaire aux programmes officiels destinés à en préciser certains contenus d'apprentissage et à programmer l'enseignement des savoirs disciplinaires selon une progression spiralaire cohérente.

${ }^{v}$ La critique littéraire fait partie des genres à dominante justificative. Chartrand, Émery-Bruneau, Sénéchal et Riverin (2013) proposent également la justification d'un problème de grammaire, la table ronde, la discussion, la critique de film et l'exposé critique. Dans le programme de français du $1^{\text {er }}$ cycle (MÉLS, 2004), on parle du commentaire critique, de la recommandation de lectures et d'activités culturelles, de la lettre de sollicitation ou de mise en candidature aux élections scolaires (p. 112).

${ }^{v i}$ Ce type de justification peut être considéré comme le procédé de base de l'argumentation (Golder, 1996; Gombert, 2003) qui articule d'autres procédés argumentatifs entre eux comme la concession, l'opposition ou la réfutation.

${ }^{\text {vii }}$ La transposition didactique interne correspond au passage du savoir à enseigner, tel que présenté dans les instructions officielles ou les manuels et les ouvrages didactiques de référence, au savoir effectivement enseigné en classe, qui passera finalement au stade savoir appris (Gauvin et Boivin, 2012).

viii Par exemple, l'exposé critique ou la participation à une table ronde, l'écriture de lettres de recommandation ou de notes critiques.
}

\section{Référence Auteur:}

Marie-Hélène Forget est diplômée de l'Université de Sherbrooke. Elle a réalisé un doctorat en didactique du français (écriture) portant sur les conduites de justifications d'élèves plurilingues. Elle poursuit un stage postdoctoral en didactique intégrée des langues et oriente ses recherches vers l'oral pour apprendre. Elle a aussi occupé des fonctions de conseillère pédagogique.

E-mail: forget.marie-helene@uqam.ca

Pour citer cet article:

Forget, M-H. (2015). La conduite de justification: un savoir à transposer des usages. Bellaterra Journal of Teaching \& Learning Language \& Literature, 8(1), 46-59. http://dx.doi.org/10.5565/rev/jtl3.588

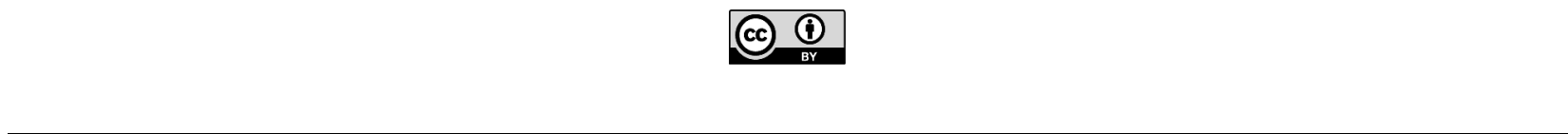

Bellaterra Journal of Teaching \& Learning Language \& Literature. 8.1 (Feb-Mar 2015)

ISSN 2013-6196 